
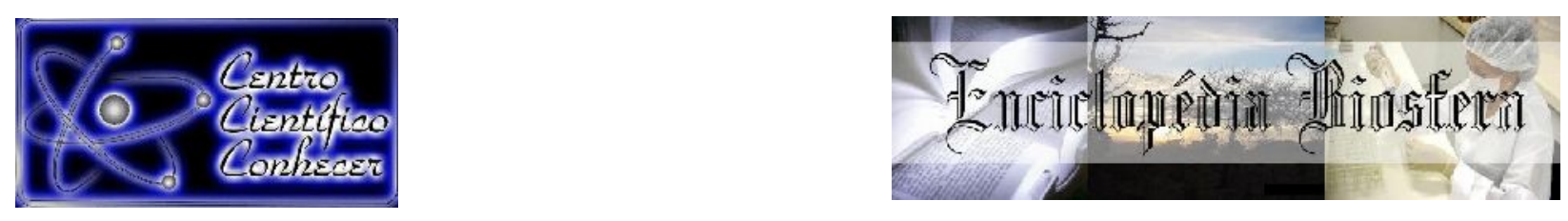

\title{
SEMILLAS DE Brachiaria ruziziensis EN DIFERENTES TIEMPOS Y NIVELES DE MEZCLA CON UREA PARA LA FORMACIÓN DE PASTO
}

Roberta Santos Santiago Amaral ${ }^{1}$, Danilo Cordeiro Maciel ${ }^{2}$, Laís Lopes Pereira ${ }^{2}$, Alexandre Martins Abdão dos Passos ${ }^{3}$, Dea Alecia Martins Netto ${ }^{3}$

1 Estudiante de Ciencias Biológicas UNIFEMM, Sete Lagoas - MG, Brasil, Becario Embrapa

2 Estudiante de Agronomía de la Universidad Federal de São João Del-Rei, Sete Lagoas - MG, Brasil, becarios del PIBIC CNPq/Embrapa

3 Investigadores de Embrapa Maíz y Sorgo - Sete Lagoas (MG) - Brasil. E-mail: alexandre.abdao@embrapa.br

Recebido em: 04/10/2019 - Aprovado em: 30/11/2019 - Publicado em: 15/12/2019 DOI: 10.18677/EnciBio_2019B53

\begin{abstract}
El cultivo de brachiaria se ha utilizado ampliamente para la implantación de áreas de pasto en sistemas integrados, utilizando fertilización con nitrógeno retardada bajo cubierta para la siembra de maíz. Sin embargo, la mezcla de fertilizantes como la urea promueve una disminución en la calidad fisiológica de la semilla con el tiempo de mezcla. El objetivo de este trabajo fue evaluar los períodos de exposición de las semillas de Brachiaria ruziziensis al contacto prolongado con urea, en tres proporciones de mezcla con fertilizante bajo la viabilidad de la semilla y el vigor de las plántulas. En un experimento de diseño completamente al azar, se evaluó el efecto de seis tiempos $(3,6,12,24,48$ y 72 horas) a tres niveles de urea $(50,100$ y $150 \mathrm{~kg}$ de $\mathrm{N} \mathrm{ha}^{-1}$ ) sobre la calidad fisiológica de las semillas. Se verificó el efecto negativo del tiempo y las proporciones de fertilizantes sobre la germinación y el vigor de las semillas. El mayor efecto negativo de la exposición de las semillas a la urea, con aumentos en la inviabilidad de las semillas y disminución de las plántulas normales, ocurre en altas proporciones de fertilizante con semillas y tiempo de mezcla hasta su uso.
\end{abstract}

RESUMEN

PALABRAS-LLAVE: Urochloa ruziziensis; Tecnología de semillas; calidad fisiológica.

\section{SEMENTES DE BRACHIARIA RUZIZIENSIS SOB DIFERENTES TEMPOS DE CONTATO E NÍVEIS DE MISTURA COM UREIA PARA FORMAÇÃO DE PASTAGEM}

\section{RESUMO}

A cultura da braquiária tem sido amplamente utilizada para implantação de áreas de pastagem em sistemas integrados, usando-se para sua semeadura, de forma defasada no milho, adubos nitrogenados em cobertura. Contudo, a mistura de fertilizantes, tais como a ureia, promove um declínio na qualidade fisiológica das sementes ao longo do tempo de mistura. Objetivou-se com o trabalho, avaliar períodos de exposição de sementes de Braquiária ruziziensis ao contato prolongado 
com ureia, em três proporções de mistura com o fertilizante sob a viabilidade das sementes e vigor das plântulas em canteiro de areia. Em um experimento em delineamento estatístico inteiramente casualizado avaliou-se o efeito de seis tempos $\left(3,6,12,24,48\right.$ e 72 horas) em três níveis de ureia $\left(50,100\right.$ e $150 \mathrm{~kg}$ de $\left.\mathrm{N} \mathrm{ha}^{-1}\right)$ sobre a qualidade fisiológica das sementes. Verificou-se que efeito negativo do tempo e das proporções de fertilizante sobre a germinação e vigor das sementes. $O$ maior efeito negativo da exposição das sementes à ureia, com incrementos na inviabilidade das sementes e decréscimo de plântulas normais, ocorre em altas proporções de fertilizante com a semente e do tempo da mistura até a sua utilização.

PALAVRAS-CHAVE: Urochloa ruziziensis; tecnología de sementes; qualidade fisiológica.

\section{INTRODUCCIÓN}

Los sistemas de integración cultivo-ganadera representan una alternativa de producción racional para suelos pobres, como los arenosos. Entre los tipos de sistemas integrados se encuentra la asociación de plantas graníferas o ensiladoras, como el maíz y el sorgo, en combinación con plantas forrajeras, especialmente las brachiarias para la generación de pastos o rastrojo en labranza de conservación (FREITAS et al., 2018; COSTA et al., 2018).

En estos sistemas, una estrategia de implementación consiste en el uso de mezclas de fertilizantes con las semillas forrajeras para la aplicación simultánea de los dos insumos durante o después de la siembra del cultivo principal (LIMA et al., 2010). En la segunda aplicación de los fertilizantes nitrogenados representa un momento adecuado para la siembra de brachiarias, disminuyendo el riesgo de competencia entre el cultivo principal y evitando mermas en el rendimiento del grano, o de la biomasa de cultivo principal durante el ensilado u otra forma de alimentación animal (COSTA et al., 2017; OLIVEIRA et al., 2018; MOMESSO et al., 2019).

Otro beneficio de esta práctica es el ahorro de combustible, al evitar aplicación aislada de cada insumo, ya que varios productores no cuentan con maquinaria e implementos adaptados para la siembra simultánea de semillas pequeñas (REIS et al., 2019).

No obstante, la mezcla de fertilizantes con semillas genera factores que aceleran el proceso de deterioro de la calidad fisiológica de las semillas (MATTEI et al., 2015; AVELINO et al., 2019). Entre los factores, se encuentra el tiempo de contacto de las semillas con los fertilizantes, ya que cuanto mayor es la exposición de las semillas, mayor es el proceso de pérdida de calidad fisiológica (MATEUS et al., 2007; CODOGNOTO et al., 2019). Se pueden citar otros factores, como el fertilizante utilizado y sus cualidades intrínsecas, como el nivel de salinidad, las condiciones ambientales de almacenamiento de mezcla (temperatura y humedad del aire), la calidad fisiológica y física inicial de los lotes de semillas, la presencia y calidad de la cobertura de semillas (BEWLEY et al., 2013; SILVA et al., 2018; JOHANN et al., 2019).

Sin embargo, se carece de información sobre la proporción de fertilizantes en la mezcla en relación con la masa de semillas puras y viables de alta calidad fisiológica de la brachiaria y sus posibles efectos sobre el vigor y la viabilidad. El objetivo de este estudio fue evaluar los períodos de contacto de las semillas de brachiarias en condiciones de estrés en contacto con dosis crecientes de urea en busca de siembra simultánea de la semilla con el fertilizante. 


\section{MATERIALES Y MÉTODOS}

En un diseño estadístico completamente aleatorio, se evaluó el efecto de seis tiempos de contacto $(3,6,12,24,48$ y 72 horas) de semillas de Brachiaria ruziziensis en tres proporciones de mezcla de urea $(50,100$ y $150 \mathrm{~kg}$ de N/ha) sobre la viabilidad (germinación) y vigor de las plántulas. Además de un tratamiento adicional, que incluía las semillas de brachiarias sin exposición al fertilizante como tratamiento de control (testigo) en esquema factorial con tratamiento adicional.

En la prueba de germinación, la siembra se realizó en charolas que contenían suelo arenoso con unos $16 \%$ de arcilla retirados del potrero con avanzada degradación agronómica y biológica. Las semillas también se colocaron en cajas gerboxen dos hojas de papel en germinadores con fotoperíodo y temperaturas controladas específicas para la especie evaluada $\left(35^{\circ} \mathrm{C}\right.$ para 8 horas de radiación y $20{ }^{\circ} \mathrm{C}$ para 16 horas en ausencia de luz). La parcela experimental constaba de cincuenta semillas puras viables, previamente separadas manualmente. La separación de las semillas del fertilizante se realizó por el método húmedo después de los períodos de contacto evaluados, utilizando agua desionizada y agitador magnético. Las semillas fueron posteriormente retiradas del agua por medio de un tamiz y secadas sobre papel toalla bajo condiciones controladas $\left(25^{\circ} \mathrm{C}\right)$. Las proporciones utilizadas se tomó como referencia la aplicación de 3 niveles de fertilizantes, recomendados para diversas regiones y condiciones de cultivo del maíz, que comprenden $\left(50,100\right.$ y $150 \mathrm{~kg}$ de $\left.\mathrm{N} \mathrm{ha}^{-1}\right)$. Para la definición de las proporciones se utilizó una tasa de siembra de 320 puntos valor cultural (PVC), siendo semillas, desnudas y sin tratamiento, presentando un $90 \%$ de valor cultural.

Se utilizó un fertilizante nitrogenado, urea, que contiene un $46 \%$ de $\mathrm{N}$, presentando un índice de salinidad del $76 \%$ en relación con el nitrato de sodio. Se usó una masa de agua que comprende 2,5 veces la del papel en las pruebas de germinación. Mientras que para la evaluación del vigor se utilizó sustrato de suelo arenoso, se realizó la determinación de la capacidad de campo del suelo $\left(0,17 \mathrm{dm}^{3}\right.$ $\mathrm{dm}^{-3}$ ) y la retención de agua antes del experimento para determinar el volumen de agua a aplicar en siembra (60\% de la capacidad de campo) y su reposición hasta la evaluación. Las bandejas se colocaron en una cámara de crecimiento simulando las temperaturas nocturnas de $20 \stackrel{\circ}{\circ} \mathrm{C}$ y diurnas de $35 \stackrel{\circ}{\circ} \mathrm{C}$, adecuadas para las brachiarias.

Se realizaron lecturas después de 21 días para determinar el número de plántulas emergentes y semillas muertas, plántulas anormales y normales en el ensayo de germinación (BRASIL, 2009). Posteriormente, los valores obtenidos se transformaron en porcentajes. Las plántulas anormales fueron las que no tenían todas las estructuras primarias, desproporciones entre ellas y una longitud aérea inferior a $20 \mathrm{~mm}$. Las plántulas normales presentaron radículas y partes aéreas completamente desarrolladas. La eficacia agronómica de los tratamientos se verificó mediante la relación entre los valores obtenidos para la germinación y la emergencia en el lecho en relación con el tratamiento adicional, testigo, sin la adición de urea (testigo).

Las suposiciones para ANOVA fueron verificadas por las pruebas ShapiroWilk y Levene. Se aplicó el análisis de varianza, seguido por la prueba de medias de Scott-Knott y las interacciones por regresiones y respuesta superficial. 


\section{RESULTADOS Y DISCUSIÓN}

Se observa el comportamiento sobre la viabilidad y vigor de las brachiarias asociados a los factores (Cuadro 1). Entre las interacciones, el efecto sólo se observó en la proporción de semillas anormales (Cuadro 1). Los tratamientos adicionales (testigo) difirieron para la emergencia de las semillas arena.

CUADRO 1 - Resumen de ANOVA para la emergencia de arena, porcentaje de germinación de plántulas normales, anormales y semillas de Brachiaria ruziziensis muertas en diferentes tiempos de contacto en dosis de urea para siembra en sistemas de Santa Fe.

\begin{tabular}{|c|c|c|c|c|c|}
\hline Causas de Cambio & GL & & Cuadrados medios & & \\
\hline & & Emergenciaa & Normales & Anormales & Muertas \\
\hline Nitrógeno (N) & 2 & $847,27^{*}$ & $798,00^{* \star}$ & $9,39^{N S}$ & $976,39^{\star \star}$ \\
\hline Tiempo (T) & 5 & $232,55^{\mathrm{NS}}$ & $1131,30^{* *}$ & $41,65^{\text {NS }}$ & $1493^{* *}$ \\
\hline $\mathrm{N} \times \mathrm{T}$ & 10 & $281,02^{\text {NS }}$ & $101,8^{N S}$ & $80,99^{* \star}$ & $69,86^{\mathrm{NS}}$ \\
\hline (Factorial) & 17 & $333,38^{*}$ & $486,50^{* *}$ & $60,99^{* *}$ & $595,24^{\star *}$ \\
\hline Adicto. vs. Factorial & 1 & $3375,56^{* *}$ & $73,92^{\text {NS }}$ & $23,16^{\mathrm{NS}}$ & $179,83^{\text {NS }}$ \\
\hline (Tratamientos) & 18 & $502,39^{\star *}$ & $463,58^{\star *}$ & $58,89^{\star *}$ & $572,16^{\star *}$ \\
\hline Residuos & 57 & 164,19 & 120,44 & 19,68 & 156,51 \\
\hline Total & 75 & & & & \\
\hline
\end{tabular}

Los parámetros medidos, plántulas normales y emergencia, fueron mayores para las menores proporciones de urea com la Brachiaria ruziziensis (Cuadro 2). A su vez, el aumento en la proporción de fertilizantes nitrogenados, especialmente en su nivel más alto, disminuyó tanto número de semillas muertas. La pérdida de potencial de germinación se ha encontrado en la literatura para varias especies de brachiarias en contacto con fertilizantes (SILVA et al., 2009; PERES et al., 2012; LIMA et al., 2014; CODOGNOTO et al., 2019).

CUADRO 2 - Promedios de emergencia de arena, porcentaje de germinación de plántulas normales, anormales y semillas de Brachiaria ruziziensis muertas y niveles de urea en la mezcla de semillas: urea

\begin{tabular}{lllllll}
\hline $\begin{array}{l}\text { Dosis } \\
\mathbf{( N ~ h a ~}^{-1} \text { ) }\end{array}$ & $\begin{array}{l}\text { Normales } \\
(\%)\end{array}$ & $\begin{array}{l}\text { Anormales } \\
(\%)\end{array}$ & $\begin{array}{l}\text { Muertas } \\
(\%)\end{array}$ & $\begin{array}{l}\text { Emergencia } \\
(\%)\end{array}$ \\
\hline $\mathbf{5 0}$ & 39,08 & a & 8,75 & 52,18 & $\mathrm{~b}$ & $58,77 \mathrm{a}$ \\
$\mathbf{1 0 0}$ & 30,58 & $\mathrm{~b}$ & 7,66 & 61,75 & $\mathrm{a}$ & $47,33 \mathrm{~b}$ \\
$\mathbf{1 5 0}$ & 28,08 & $\mathrm{~b}$ & 7,66 & 64,25 & $\mathrm{a}$ & $46,50 \mathrm{~b}$ \\
\hline Média & 32,58 & & 8,02 & 59,39 & & 50,87 \\
\hline
\end{tabular}

Los promedios seguidos de las mismas letras son estadísticamente iguales (Scott Knott $(p<0.05))$.

A partir de la respuesta promedio de pérdida de vigor en función al tiempo de contacto con la urea de Brachiaria ruziziensis (Figura 1), se observa que a partir de las 3 horas de contacto las semillas sufren una disminución media de aproximadamente el $26,3 \%$, acercándose a una emergencia media del $50 \%$ a partir del $68,8 \%$ observados con un tiempo de exposición de 3 horas. Las plantas forrajeras sometidas a diferentes tiempos de exposición a fertilizantes minerales ENCICLOPÉDIA BIOSFERA, Centro Científico Conhecer - Goiânia, v.16 n.30; p.586 
(MATEUS et al., 2007; DAN et al., 2011; MATTEl et al., 2019) se informan en la literatura que muestran pérdidas significativas de vigor de las plántulas. Sin embargo, no existen estudios que muestren variaciones tales como los niveles de fertilizantes utilizados en la mezcla, especialmente utilizando semillas de alto nivel de calidad fisiológica con un valor cultural igual o superior al $90 \%$.

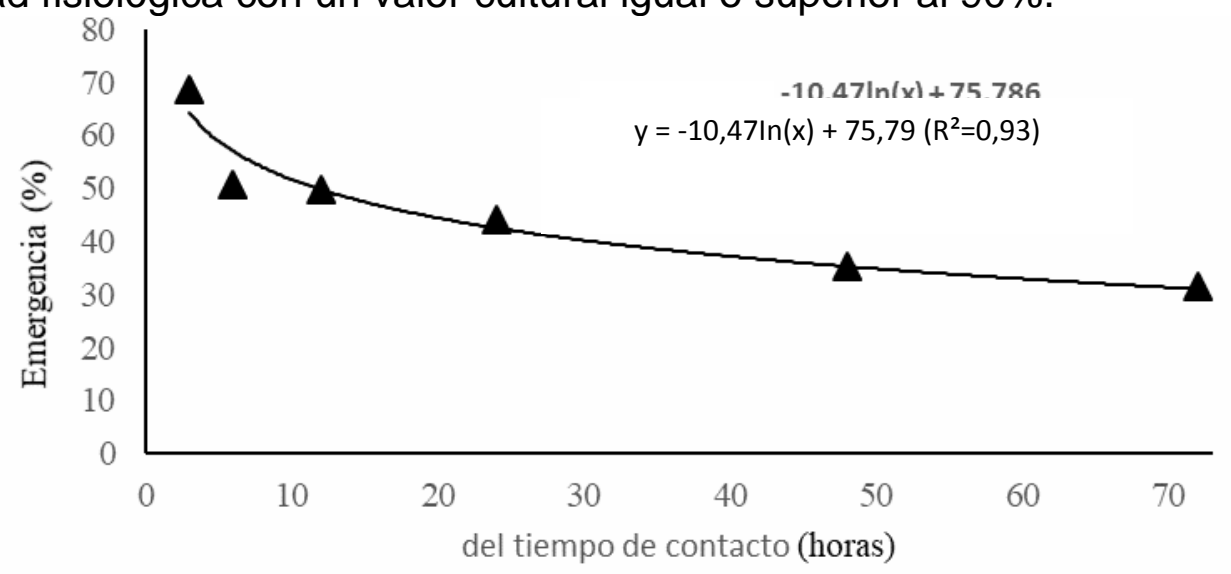

FIGURA 1 - Pérdida de vigor (\%) en función del tiempo de contacto (h) del fertilizante en la Brachiaria ruziziensis. Fuente: los autores.

Se observó un efecto deplectivo sobre la germinación y el vigor de las semillas en función del tiempo de contacto de las semillas con el fertilizante nitrogenado y en relación con la proporción de mezcla de semillas y urea (Figura 2). Ambos factores analizados (urea y tiempo) aumentaron el porcentaje de semillas no germinadas (muertas), y la disminución en el porcentaje de plántulas normales.

Ampliación $(\%)=10,089+1,709 x+1,562 y-0,0071 x^{*} x+0,0055 x^{*} y-0,008 y^{*} y$

Decrecimiento $(\%)=30,98+0,1296 x+0,4381 y$

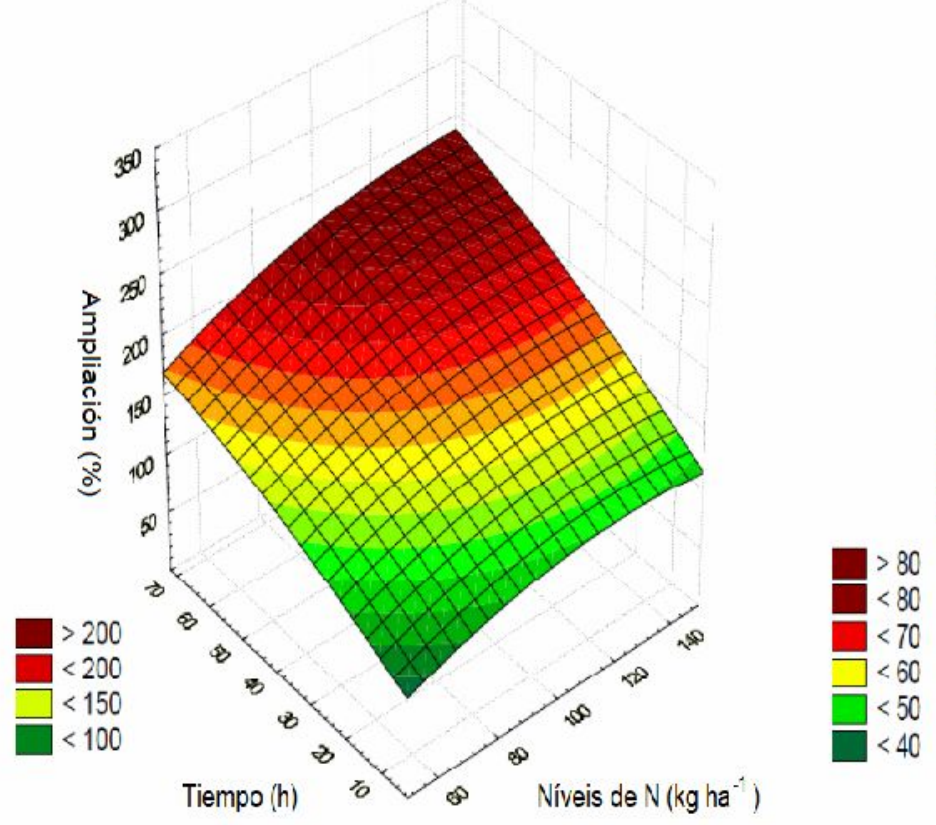

MUERTAS

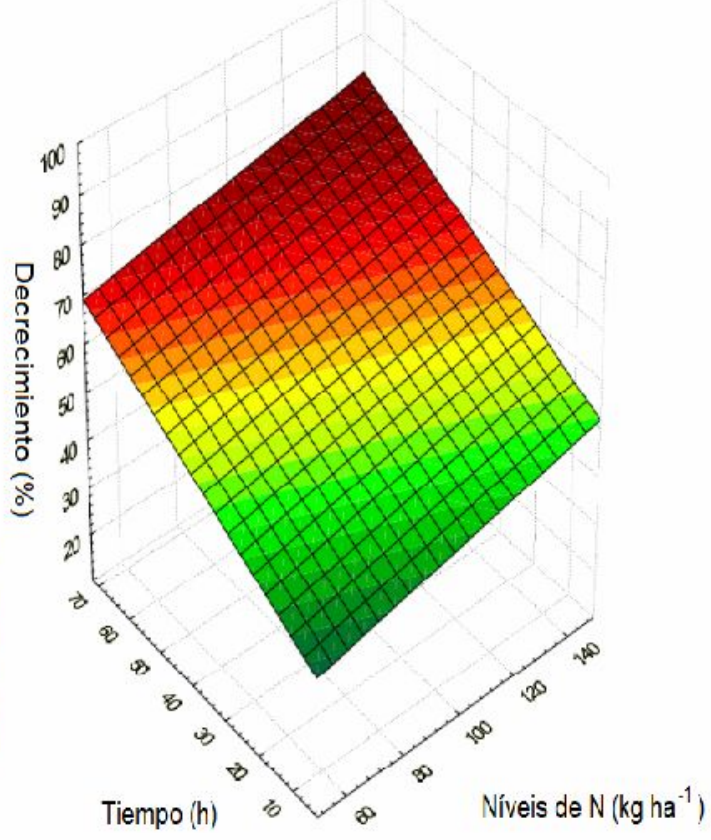

NORIMALES

FIGURA 2 - Superficies de respuesta en el aumento de porcentaje de semillas muertas (A) y plántulas normales (B) por la combinación de dosis de nitrógeno y tiempos de contacto entre las semillas de Brachiaria ruziziensis y la urea. Fuente: los autores. 
Las semillas de Brachiaria ruziziensis demostró susceptibilidad al aumento en la proporción entre la urea y las semillas, con disminución lineal por cada incremento en las variables independientes evaluadas. Resultado similar ha encontrado en Codognoto et al. (2019), quienes observaron pérdidas lineales en la emergencia de las brachiarias. Dan et al. (2011) evaluaron semillas de Brachiaria ruziziensis con menor potencial de germinación y pureza, pero observaron pérdidas lineales en el vigor de las semillas de Brachiaria ruziziensis debido al mayor contacto de la semilla con la urea.

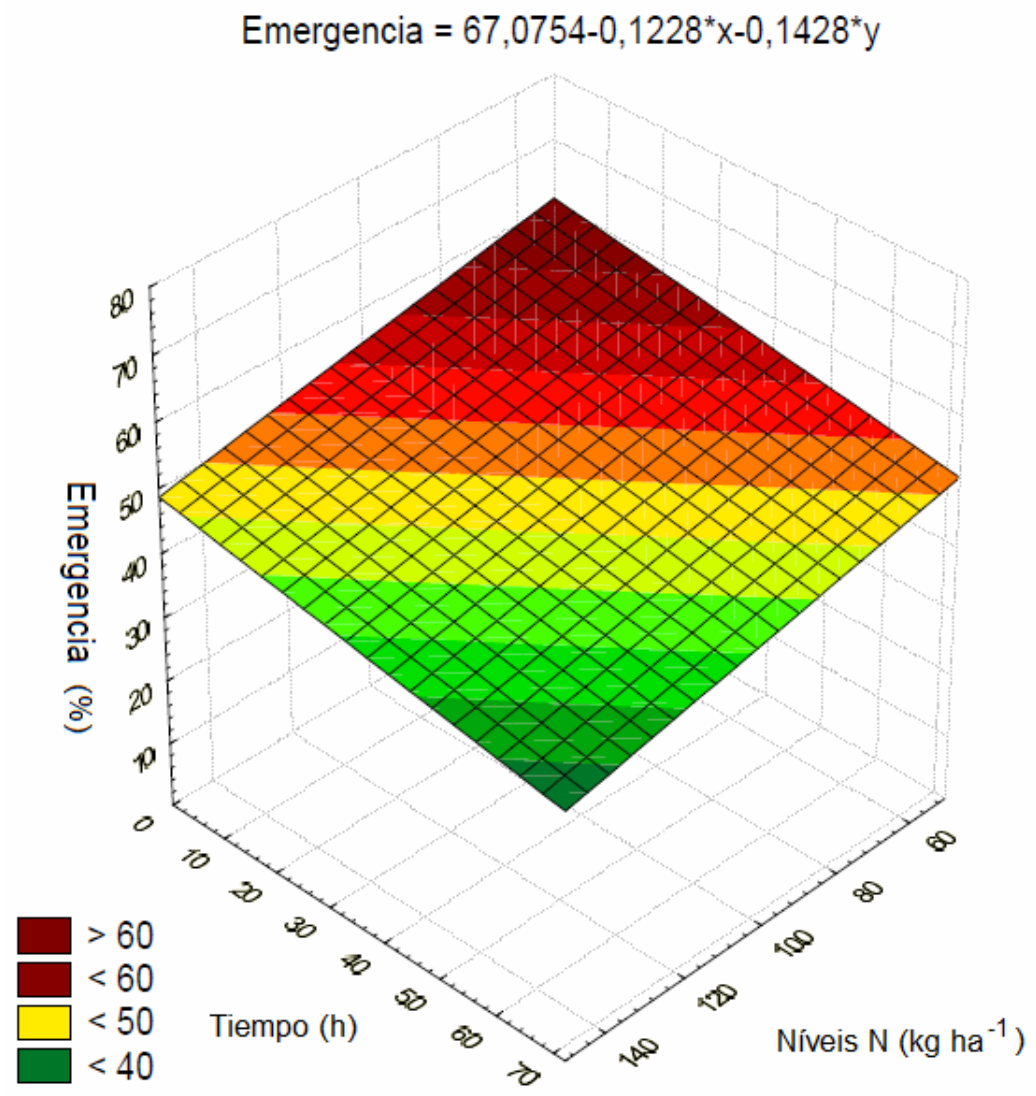

FIGURA 3 - Regresión para la emergencia de las Brachiaria ruziziensis en arena en diferentes dosis de nitrógeno y tiempos de contacto con la urea. Fuente: los autores.

La baja calidad fisiológica y física de las semillas es un factor limitante en la formación de áreas de pastoreo en Brasil (SILVA et al., 2019). En el país, los pastizales han mostrado altos niveles de degradación y la integración de la ganadería es una alternativa sostenible para la recuperación de estas áreas (VALLE JUNIOR et al., 2019), generando su eficiente reincorporación en el proceso productivo (REIS et al., 2019; TORRES et al., 2019). Por lo tanto, el uso de semillas de alta calidad fisiológica es una premisa para la profesionalización y mejora del sector.

\section{CONCLUSIONES}

La pérdida de vigor en el establecimiento inicial ocurre logarítmicamente, de la Brachiaria ruziziensis se recomienda el uso de la mezcla dentro de las primeras tres horas de contacto en condiciones ambientales amenas de 25ํㅡ de almacenamiento del conjunto fertilizante /semilla de alta calidad fisiológica. 
Existe un mayor efecto negativo de la exposición de la semilla a la urea, con aumentos en la inviabilidad de la semilla y disminución de las plántulas normales, mayor es la relación fertilizante / semilla y el tiempo de mezcla hasta su uso.

\section{REFERENCIAS}

AVELINO, A.; FARIA, D.; CABRAL, C.; ABREU, J.; CAMILI, E.; SALES, K.; \& BUNGESTAB, D. Maximum contact time of "Piatã Grass" Brachiaria brizantha (Poaceae) seeds with fertilizer for germination. Journal of Experimental Agriculture International, v. 35, n. 1, p. 1-9, jul. 2019. Disponible en: <http://dx.doi.org/10.9734/JEAl/2019/v35i130193>. 10.9734/JEAI/2019/v35i130193

BEWLEY, J. D., BRADFORD, K. J., HILHORST, H. W. M., Y NONOGAKI, H. (2013). Seeds: Physiology of Development, Germination and Dormancy. New York, NY: Springer. Disponible en:

https://www.researchgate.net/publication/285948358_Seeds_Physiology_of_develop ment_germination_and_dormancy_3rd_edition> doi: 10.1007 / 978-1-4614-4693-4

BRASIL. Ministério da Agricultura, Pecuária e Abastecimento. Regras para análise de sementes. Brasília, 2009. 399 p.

CODOGNOTO, L.; CONDE, T.; MALTONI, K.; FARIA, G.; REIS, A. Physiological response of marandu grass seeds exposed to NPK fertilizer. Ciência Rural, v. 49:06.2019. Disponible en: <http://dx.doi.org/10.1590/0103-8478cr20180084>. doi: $10.1590 / 0103-8478 c r 20180084$

COSTA, N.R.; ANDREOTTI, M.; CRUSCIOL, C.A.C.; LIMA, C. G. R.; CASTILHOS, A. M.; et al. Yield and nutritive value of the silage of corn intercropped with tropical perennial grasses. Pesquisa agropecuária brasileira (online), v. 52, p. 63-73, 2017. Disponible em: <http://dx.doi.org/10.1590/s0100-204x2017000100008>. doi: 10.1590/s0100-204×2017000100008

COSTA, K. A. P.; SOUZA, W. F.; SILVA, V. C.; EPIFANIO, P. S.; SANTOS, H. S. Protein and carbohydrates fractionation in Paiaguas palisadegrass intercropped with grain sorghum in pasture recovery. Acta Scientiarum. Animal Sciences, v. 41, e. 42693, 2018.

Disponible

en: <https://dx.doi.org/10.4025/actascianimsci.v41i1.42693.> doi: 10.4025/actascianimsci.v41i1.42693

DA SILVA, D.; DE OLIVEIRA, G.; RAGONHA, E.; \& RAGONHA, E. Superfosfato triplo como via de distribuição de sementes de Brachiaria brizantha para renovação de pastagens na Amazônia. Revista Brasileira de Zootecnia. v.38, n.5, p.796-800, 2009. Disponible en: <http://dx.doi.org/10.1590/S1516-35982009000500003>. doi:10.1590/S1516-35982009000500003

DAN, H. A.; DAN, L. G. M.; BARROSO, A. L. L.; BRACCINI, A.L.; PICCININ, G.G. Mistura de sementes de Brachiaria ruziziensis com ureia visando à implantação do sistema de Integração Lavoura-Pecuária. Revista Caatinga (UFERSA), v.24, n.3, 
p.68-73,

2011.

Disponible

en:

<https://periodicos.ufersa.edu.br/index.php/caatinga/article/view/2088>

FREITAS, M. A. M.; SILVA, D. V.; GUIMARÃES, F. R.; LEAL, P. L.; SOUZA, F. M. M.; et al. Biological attributes of soil cultivated with corn intercropped with Urochloa brizantha in different plant arrangements with and without herbicide application. Agriculture ecosystems \& environment, v. 254, p. 35-40, 2018. Disponible en: <https://doi.org/10.1016/j.agee.2017.10.026>. doi: 10.1016/j.agee.2017.10.026

JOHANN, A. K.; BIGOLIN, G.; HARTER, L.S.H. Avaliação da qualidade fisiológica de semntes de linho (Linum usitatissimum L.) pelo teste de envelhecimento acelerado. Enciclopédia Biosfera, Centro Científico Conhecer- Goiânia, v.16 n.29; p. 323-338. 2019.

<http://www.conhecer.org.br/enciclop/2019a/agrar/avaliacao\%20da\%20qualidade.pdf >.doi: 10.18677/EnciBio_2019A24 
LIMA, E.; TAVARES, J.; AZEVEDO, V.; \& LEITÃO-LIMA, P. Mistura de sementes de Brachiaria brizantha com fertilizante NPK. Ciência Rural, v. 40, p.441-444. 2010. Disponible en: < http://dx.doi.org/10.1590/S0103-84782010005000003>. doi: 10.1590/S0103-84782010005000003

LIMA, E.; TAVARES, J.; LEITÃO-LIMA, P.; PINHEIRO, D.; \& SANTOS, W. Germinação e vigor de sementes de Brachiaria brizantha cv. Marandú em função do tempo de mistura com uréia. Revista de Agricultura, v. 89, p. 41-52. 2014. Disponible $<$ http://www.fealq.org.br/ojs/index.php/revistadeagricultura/article/view/63> en:

MATTEI, E.; ACHRE, D.; MATTIA, V.; YASSUE, R. M.; BOSQUETTE, W. R.; et al. Seed germination and development of Brachiaria ruziziensis after different periods of contact with fertilizers. Journal of Agronomic Sciences, v.4, n.2, p.20-30, 2015. Disponible en: <http://www.dca.uem.br/V4N2/03-Eloisa.pdf>

MATEUS, G.; BORGHI, E.; MARQUES, R.; BÔAS, R.; \& CRUSCIOL, C. Fontes e períodos de contato de fertilizantes e germinação de sementes de Brachiaria brizantha. Revista Brasileira de Ciência do Solo, v. 31, p. 177-183. 2007. Disponible en: <http://dx.doi.org/10.1590/S0100-06832007000100018>. doi: 10.1590/S0100-06832007000100018

MOMESSO, L.; CRUSCIOL, C. A.C.; SORATTO, R. P.; VYN, T. J.; TANAKA, K. S.; et al. Impacts of Nitrogen Management on No-Till Maize Production Following Forage Cover Crops. Agronomy journal, v. 111, p. 2, 2019. Disponível em <dx.doi.org/10.2134/agronj2018.03.0201>. doi: 10.2134/agronj2018.03.0201

OLIVEIRA, R.J; BRUGNARA, A.S.; ADAMI, P.F.; GLIENKE, L.C.; JUNIOR, A.A.B. Corn and alexandergrass intercropping system: influences of herbicide management on grain and forage yield. Colloquium agrariae (UNOESTE), v. 14, p. 66-72, 2018. Disponible en: < http://journal.unoeste.br/index.php/ca/article/view/2105/2194>

PERES, A.; VAZQUEZ, G.; \& CARDOSO, R. Physiological potential of Brachiaria brizantha cV. Marandu seeds kept in contact with phosphatic fertilizers. Revista Brasileira de Sementes, v.34, p. 424-432, 2012. Disponible en: <http://dx.doi.org/10.1590/S0101-31222012000300009>. doi: 10.1590/S010131222012000300009

SILVA, D.C.B.; OLIVEIRA, G.P.; RAGONHA, E.P. Potencial fisiológico de Convert hd364, em diferentes períodos de contato com adubo. Revista Thema, v.15, n.2. 2018. Disponible en: <http://dx.doi.org/10.15536/thema.15.2018.531-538.884>. doi: 10.15536/thema.15.2018.531-538.884

SILVA, G. Z. DA; MARTINS, C. C. CRUZ; J. O. JEROMINI; T. S. \& MACHADO, C. G. Regiões de produção e qualidade física de sementes de Urochloa decumbens CV. Basilisk. Bioscience Journal, v. 35, 2019. Disponible en: <https://doi.org/10.14393/BJ-v35n1a2019-41749>. doi: 10.14393/BJ-v35n1a201941749 
REIS, J. C.; KAMOI, M. Y. T.; LATORRACA, D.; CHEN, R. F. F.; MICHETTI, M.; et al. Assessing the economic viability of integrated crop-livestock systems in Mato Grosso, Brazil. Renewable agriculture and food systems, v. 1, p. 1-12, 2019. Disponible en: <https://doi.org/10.1017/S1742170519000280>. doi: 10.1017/S1742170519000280

VALLE JÚNIOR, R.F.; SIQUEIRA, H. E.; VALERA, C. A.; OLIVEIRA, C. F; SANCHES, L. F. F. et al. Diagnosis of degraded pastures using an improved NDVIbased remote sensing approach: An application to the Environmental Protection Area of Uberaba River Basin (Minas Gerais, Brazil). Remote Sensing Applications: Society and Environment, v. 14, p. 20-33, 2019. Disponible en: <https://doi.org/10.1016/j.rsase.2019.02.001>. doi: 10.1016/j.rsase.2019.02.001

TORRES, F. N.; RICHTER, R.; VOHLAND, M.A. A Multisensoral approach for highresolution land cover and pasture degradation mapping in the humid tropics. A case study of the fragmented landscape of Rio de Janeiro. International Journal of Applied Earth Observation and Geoinformation, v.78, p. 189-201, 2019. doi 03032434. Disponible en: <https://doi.org/10.1016/j.jag.2019.01.011>. doi: 10.1016/j.jag.2019.01.011 